\title{
Articulación entre la NTC-ISO 9001:2015 y los lineamientos de acreditación de programas del CNA en Unitrópico*
}

\author{
Articulation between NTC-ISO 9001:2015 and the program \\ accreditation guidelines of CNA in Unitrópico
}

\section{União entre a NTC-ISO 9001:2015 e as diretrizes de credenciamento de programas do CNA em Unitrópico}

Recibido: 14 de agosto de 2018 Revisado: 15 de septiembre de 2018

Aceptado: 1 de marzo de 2019

Fernando Augusto Trujillo Suáre***

Fundación Universitaria Internacional del Trópico Americano-Unitrópico, Colombia

Ximena Lucía Pedraæa Nájar****

Universidad Santo Tomás, Colombia

Cómo citar: Trujillo-Suárez, F. A. y Pedraza-Nájar, X. L. (2019). Articulación entre la NTC-ISO 9001:2015 y los lineamientos de acreditación de programas del CNA en Unitrópico. Signos. Investigación en Sistemas de Gestión, 11(2), 119-130. Doi: https://doi.org/10.15332/24631140.5085

* Artículo de investigación.

** Magíster en Calidad y Gestión Integral, ingeniero de Alimentos. Yopal, Colombia. Correo electrónico: fernando.trujillo@unitropico.edu.co, Orcid: https://orcid.org/0000-0002-5879-2114

*** Doctora en Administración, magíster en Calidad y Gestión Integral, especialista en Gestión de la Producción, la Calidad y la Tecnología; especialista en Gerencia de Procesos, Calidad e Innovación, microbióloga. Bogotá, Colombia. Correo electrónico: ximenapedraza@usantotomas.edu.co. Orcid: https://orcid.org/0000-0003-0414-0636 


\section{RESUMEN}

El sistema educativo colombiano en las IES (instituciones de educación superior) toma como pilar fundamental la integralidad del individuo para permitirle defender y demandar el respeto a una vida digna, exigir sus derechos y cumplir con sus deberes. Unitrópico proyecta la cultura en las artes, la ciencia, la tecnología y la educación, así como en la vida cotidiana y en la creación popular. Actualmente busca la implementación de un sistema integrado de aseguramiento de la calidad bajo los requisitos de la NTC (Norma Técnica Colombiana) ISO 9001:2015 y la acreditación de programas según los lineamientos del Consejo Nacional de Acreditación. Sin embargo, es evidente la ausencia de procesos y procedimientos orientados al logro de la eficiencia y eficacia institucional. Por esta razón, a través de un paradigma positivista y un método mixto de carácter descriptivo se busca determinar una propuesta metodológica integrada para la institución, que cumpla los requisitos de la referida norma técnica en función de mejorar la eficiencia en la prestación del servicio con la consolidación de la mejora continua en la institución. Se fortalece así la ruta hacia la acreditación institucional con un enfoque en los principios y valores de la calidad.

Palabras clave: educación superior, calidad, acreditación, integración.

\section{ABSTRACT}

The Colombian educational system in Higher Education Institutions is underpinned by integrality of individuals, allowing them to defend and demand respect for a decent life, upholding their rights and fulfilling their duties. Unitrópico promotes culture in arts, science, technology and education, as well as in everyday life and in people's creation. Nowadays, it is searching for the implementation of an integrated quality assurance system under the requirements of the Colombian Technical Standard ISO 9001:2015 and program accreditation as per the guidelines of the National Accreditation Council; however, the lack of processes and procedures oriented toward the attainment of institutional efficiency and effectiveness is evident. This is why, through a positivist paradigm employing a mixed descriptive method, an integrated methodological proposal for the institution was determined, to meet the requirements of a technical standard and the regulatory requirements to improve efficiency in service provision through the consolidation of continuous improvement at the institution toward the institutional accreditation, focusing the strengthening on quality principles and values.

Keywords: Higher Education, quality, accreditation, integration.

\section{RESUMO}

O sistema educacional colombiano nas Instituições de Ensino Superior, tem como base fundamental a integridade do indivíduo, permitindo-lhe defender e exigir o respeito a uma vida com dignidade, cobrando seus direitos e cumprindo seus deveres. Unitrópico projeta a cultura nas artes, na ciência, na tecnologia e na educação, bem como na vida quotidiana e na criação popular. Na atualidade procura a execução de um sistema integrado de garantia da qualidade sob os requisitos da Norma Técnica Colombiana ISO 9001: 2015 e o credenciamento de programas conforme as diretrizes do Conselho Nacional de Acreditação, no entanto, revelase a ausência de processos e procedimentos voltados ao sucesso de uma eficiência e eficácia institucionais. Por esse motivo, através de um paradigma positivista com um método combinado do tipo descritivo, consegue-se 
determinar uma proposta metodológica integrada para a instituição, que se propõe superar o adimplemento dos requisitos de uma norma técnica e os regulamentares para melhorar a eficiência na prestação do serviço com a consolidação da melhoria continua na instituição até a acreditação institucional com um fortalecimento focado nos princípios e valores da qualidade.

Palavras-chave: Ensino superior, qualidade, acreditação, integração.

\section{INTRODUCCIÓN}

La modernización y adaptación de la educación superior a los tiempos actuales requiere de esfuerzos inquebrantables, sistemáticos e institucionalizados de investigación y análisis permanentes con el fin de consolidar una comunidad y un pensamiento estratégico para la socialización del conocimiento en busca de generar políticas públicas orientadas hacia el proceso de aseguramiento de la calidad. En efecto, la acreditación de programas de educación superior a nivel nacional y regional exige cumplir con algunas condiciones establecidas por la ley para la obtención de un aval de operación, como el registro calificado, y reconocimientos voluntarios en calidad, como la acreditación de alta calidad de programas.

En los últimos años, autores como Powell (1995), Hendricks y Singhal (1996), Rahman y Bullock (2005) y Kumar, Choisne, De Grosbois y Kumar (2009) han podido demostrar que la gestión de la calidad es un método que permite a las organizaciones aumentar su competitividad y lograr mejores resultados en el desarrollo de sus actividades. En ese marco la cultura organizacional es uno de los factores más importantes a tener en cuenta para el éxito sostenido (o fracaso) en función de la satisfacción del cliente. Para el éxito es fundamental un desempeño estructural y procesos administrativos eficientes, lo que se logra mediante una política de recursos humanos y liderazgo. Es importante también la motivación del personal lograda mediante un buen clima organizacional, que se relaciona directamente con el rendimiento laboral, la participación, el compromiso, la motivación, la calidad, la satisfacción y la innovación. En las instituciones educativas todo eso impacta directamente en la calidad de los programas académicos y el logro de la misión, la visión y las políticas institucionales (Tapia, 2015; Espín, Jiménez y Costa, 2014).

El modelo de acreditación colombiano del CNA (Consejo Nacional de Acreditación) articula referentes universales alineados con la misión y el PEI (proyecto educativo institucional). Así, evalúa cada IES y sus programas académicos mediante factores y características asociados a criterios de calidad que garantizan las condiciones óptimas requeridas por las comunidades académicas de cada una de las profesiones o disciplinas en coherencia con la naturaleza y los objetivos de la institución. Es importante recordar que la acreditación de programas, como un requisito fundamental para la futura acreditación institucional —donde la institución desplegará sus recursos físicos y humanos para lograr el cumplimiento de su misión y visión mediante la implementación de procesos eficientes que tengan un impacto en el medio a nivel regional y nacional-, debe contar con la capacidad de sostener a largo plazo su PEI, que a su vez habrá de contener una visión clara hacia el futuro (CNA, 2016).

Unitrópico, tras reconocer que la educación superior es un servicio de enorme importancia social y estratégica para la región y el país, desarrolla funciones educativas con garantía de calidad, cumple su misión con responsabilidad social mediante la administración eficiente de los recursos y atiende a los requerimientos de la sociedad y la demanda laboral ofreciendo currículos y 
programas sólidos, además de flexibles y pertinentes (Morduchowicz y Arango, 2007). De esta manera, mediante la investigación, la proyección social y la docencia esta importante IES de la Orinoquía propende a generar conocimientos de su entorno evaluando y aprovechando su riqueza natural, su biodiversidad y su diversidad étnica con prácticas sostenibles que aportan al desarrollo económico y social de la región (Unitrópico, Consejo Superior, 2011).

Dentro de su compromiso con la sociedad y con el desarrollo del país esta IES busca, como el Estado colombiano lo exige, dar testimonio de la calidad de sus programas académicos por medio de una metodología de evaluación con la participación de la comunidad universitaria, las comunidades académicas y el CNA. Tal testimonio se basa en la autonomía y la autorregulación, que permiten a las IES rendir cuentas de sus actividades a la sociedad en un espacio de fortalecimiento de la calidad de la educación impartida. La metodología establecida, evaluada por pares externos, fortalece la calidad educativa y logra así el reconocimiento público de la institución como actor fundamental en el desarrollo departamental y nacional. La acreditación de programas no se centra en la vigilancia, el control y la inspección, sino en el fomento y la mejora continua de la educación superior (CNA, 2013).

Para mantener la continuidad de los procesos de gestión en los programas académicos de Unitrópico se deben desarrollar actividades tendientes al cumplimiento de la normatividad vigente en términos de acreditación, así como parámetros para implantar a futuro un sistema de gestión basado en procesos. Por lo tanto, se hace necesario construir una metodología que permita estandarizar dichas actividades con el fin de implementar un sistema integrado de aseguramiento de la calidad que articule la NTC-ISO 9001:2015 y los lineamientos para la acreditación de programas del
CNA. De esta forma se aportarán los elementos que permitan la certificación de los procesos institucionales (académicos y administrativos), así como la acreditación de los factores y características propios del modelo CNA. Se satisfarán así a la vez los criterios nacionales e internacionales de gestión de calidad y los principios de la acreditación de programas académicos de educación superior en Colombia.

Las ventajas de la integración de los sistemas de gestión de la calidad son evidentes en cuanto a mejorar el desarrollo y la transferencia de tecnologías, la ejecución de los procesos y actividades en la producción, los procesos internos de gestión, la motivación y enpoderamiento del personal, la eficiencia en las actividades planificadas en las auditorías, la confianza del cliente y el buen nombre de la organización, el cumplimiento de los objetivos definidos y la permanencia en el mercado (Karapetrovic y Willborn, 1998); Ferguson, García y Bornay, 2002).

En este contexto la presente investigación propone una metodología para la articulación de los requisitos de la NTC-ISO 9001:2015 y los lineamientos de acreditación de programas del CNA evaluando la posible implementación de un sistema integrado de la calidad en Unitrópico. Con ello en mente se realizó un diagnóstico de la situación actual frente al cumplimiento de los requisitos de los dos referenciales de calidad en la IES mediante la aplicación de una encuesta semiestructurada a miembros estratégicos de la comunidad universitaria. Luego se hizo un diagnóstico que permitió evaluar el porcentaje de cumplimiento de la norma versus los lineamentos de acreditación de programas. Finalmente, con el uso de la herramienta de hoja de análisis, se estableció el porcentaje de correlación entre los referenciales con el fin de estructurar y consolidar una metodología para su implementación en la institución objeto de estudio. 


\section{METODOLOGÍA}

El presente estudio es de alcance descriptivo puesto que pretende establecer las características de los eventos, sucesos o fenómenos en estudio especificando cómo son y se manifiestan sus propiedades, características, procesos o cualquier otro elemento que sea susceptible de análisis a la hora de describir lo que se investiga. Con ello se puede establecer que este estudio describe la NTC-ISO 9001:2015 y la acreditación de programas del CNA, y sus formas de relación, estableciendo cuáles son los puntos comunes que determinan la posibilidad de su articulación (Hernández, Fernández y Baptista, 2014).

El enfoque utilizado es mixto puesto que combina instrumentos cuantitativos y cualitativos para describir, predecir y explicar resultados medibles mediante el método deductivo-inductivo, que implica tanto la observación y evaluación de los fenómenos como el nivel de fundamentación de estos. Es decir, se estudia la integralidad de los sistemas involucrados yendo de conceptos universalmente aceptados a conceptos particulares o viceversa para, en este caso, identificar procesos comunes entre la NTC-ISO 9001:2015 y la acreditación de programas del CNA (Pérez, 2011; Ruiz, Borboa y Medina, 2013).

Se formularon las hipótesis descriptivas con el fin de predecir datos o valores mediante variables que se midieron o se pusieron bajo observación como explicación tentativa del fenómeno. Para determinar su veracidad o no hubo de tenerse en cuenta que no es fácil estimar precisamente lo sucedido con un fenómeno complejo (Hernández et al., 2014). La hipótesis de investigación (HI) fue: al desarrollar y aplicar una metodología para la articulación entre ISO 9001:2015 y los lineamientos para la acreditación de programas del CNA se mejorará la eficiencia en los procesos y la satisfacción del cliente. La hipótesis nula (H0) fue: al desarrollar y aplicar una metodología para la articulación entre ISO 9001:2015 y los lineamientos para la acreditación de programas del CNA no se mejorará la eficiencia en los procesos ni la satisfacción del cliente.

Se elaboró una entrevista semiestructurada para evaluar la conformidad con los referenciales de calidad articulados a los programas de Unitrópico, la cual se aplicó al personal estratégico de la comunidad universitaria. Esto permitió determinar el grado de conocimiento de los procesos, su importancia y la aceptación de los sistemas integrados de gestión. Por otro lado, se evaluó mediante matrices de diagnóstico y de análisis el cumplimiento en Unitrópico de los parámetros de la norma ISO 9001:2015 y su correlación con los factores y características de la acreditación de programas del CNA.

La entrevista semiestructurada se aplicó a quince miembros de la institución con responsabilidades y mando en cada una de las dependencias representadas en la estructura orgánica de Unitrópico (Unitrópico, 2011). Por el tamaño de la población no fue necesario desarrollar un muestreo. Además, se incluyó personal con cargos de autoridad en la institución que se consideran de carácter estratégico para el cumplimiento y desarrollo de especificaciones y estándares de calidad.

La entrevista tenía once preguntas de carácter abierto y de libre expresión, que permitieron determinar el conocimiento, la importancia y la pertinencia del sistema integrado de gestión de cada uno de los miembros de la comunidad Unitropista indagados. Cada respuesta fue evaluada por un grupo de expertos compuesto por tres profesionales con amplia experiencia y formación en temas de calidad o educación. Se utilizó una escala Likert para la calificación con una valoración de 1 a 5 , de la siguiente manera: 1 (nulo), 2 (deficiente), 3 (regular), 4 (bueno) y 5 (excelente) (Quintero, 2007).

Posteriormente se realizó un diagnóstico para establecer el nivel de aplicación de la estructura de alto nivel 
que define la ISO para sus sistemas de gestión desde 2012. Para eso se usó una matriz que permite evaluar la implementación de los diez capítulos de la norma de calidad ISO 9001:2015 (Icontec, 2015) estableciendo la condición de los parámetros manifestados en dicho documento: no aplicado, aplicado sin evidencias, aplicado en desarrollo, implementado, implementadoauditado o en mejora continua. La matriz permite evaluar una única opción con un valor de uno (1) en el caso que sea conforme para cada numeral de la norma o cero (0) si no es conforme. Adicionalmente se solicitó que se describieran el tipo de evidencia, los responsables, el estado de avance y los puntos críticos pendientes (Univalle, s. f.). Para complementar la fase de diagnóstico se hizo una revisión del informe de autoevaluación realizado en Unitrópico según los parámetros del CNA, que muestra el comparativo entre los dos últimos procesos de autoevaluación de la institución (periodos 20112012 y 2013-2014).

Con el fin de establecer el nivel de correlación entre la norma ISO 9001:2015 y los lineamientos de la acreditación de programas académicos del CNA se propuso una matriz basada en la herramienta de calidad de hoja de análisis presentada por Joseph Juran, que propone comparar y valorar ítems. Para este estudio se identificaron 10 factores de calidad y 40 características según lineamientos del CNA versus los capítulos 4 al 10 de la norma ISO 9001:2015, y se procedió a establecer el nivel de correspondencia o no correspondencia ítem por ítem entre cada grupo (Juran, 1990; López, 2014).

Adicionalmente se asignó una ponderación en porcentaje a cada uno de los factores y los ítems de la norma ISO 9001:2015 y se estableció tanto para los ítems de esta como para los factores de acreditación el nivel de correlación que presentan entre sí. La matriz permite la valoración mediante una escala que tiene una ponderación de la siguiente forma: 1 si el ítem no presenta correlación, 3 si la correlación es media y 5 si la correlación es alta. Así es posible obtener sumatorias por ítem, característica, factor y numeral principal, que son llevadas finalmente a valores porcentuales.

\section{RESULTADOS Y DISCUSIÓN}

En la indagación documental se logró la identificación de fundamentos teóricos y conceptuales a partir de fuentes primarias de consulta, de donde se tomó la información necesaria para realizar las entrevistas semiestructuradas, seguidas de la revisión de documentos institucionales y el trabajo de campo de verificación de condiciones de calidad en Unitrópico. Además, se recopiló y analizó información de tipo secundario en artículos de revistas científicas y publicaciones seriadas, libros, normas técnicas, sitios y páginas web y legislación nacional (Wigodski, 2010).

De otra parte, se evidencia que la IES Unitrópico en el primer proceso de autoevaluación de sus programas, entre 2011 y 2012, tiene un promedio del $47.97 \%$, lo que indica que cumple insatisfactoriamente con los estándares de acreditación; y para el periodo comprendido entre 2013 y 2014 presenta un valor porcentual del $75.6 \%$, cumpliéndolos aceptablemente. Valga señalarlo, se presentó una mejora en el cumplimiento de los diez factores de calidad según parámetros del CNA (Unitrópico, 2014).

Con respecto a las once temáticas evaluadas a través de las quince entrevistas semiestructuradas, los resultados se resumen en la tabla 1. 
Tabla 1. Temáticas y respuestas de las entrevistas semiestructuradas

\begin{tabular}{|c|c|}
\hline Temática en la entrevista & Orientación de la respuesta \\
\hline Evaluación sobre el conocimiento de la NTC-ISO 9001:2015 & $\begin{array}{l}\text { El } 73 \% \text { de los entrevistados posee conocimientos sobre la NTC-ISO } \\
\text { 9001:2015 y los lineamientos de acreditación del CNA ya que el promedio } \\
\text { del conocimiento es de } 3.22 \text { +/- } 0.6 \text { con una confianza del } 95 \%\end{array}$ \\
\hline $\begin{array}{l}\text { Evaluación sobre el reconocimiento de la articulación de los } \\
\text { sistemas estudiados }\end{array}$ & $\begin{array}{l}\text { Los entrevistados tienen un reconocimiento regular de tal articulación } \\
\text { puesto que las respuestas presentan una distribución normal, con promedio } \\
\text { de } 2.71+/-0.6 \text { con una confianza del } 95 \%\end{array}$ \\
\hline Análisis exploratorio de las ventajas competitivas de la articulación & $\begin{array}{l}\text { Los entrevistados reconocen las ventajas competitivas de la articulación } \\
\text { de la norma NTC-ISO 9001:2015 y los lineamientos de acreditación de } \\
\text { programas del CNA ya que la distribución de frecuencia es uniforme }\end{array}$ \\
\hline $\begin{array}{l}\text { Evaluación sobre la relación directa con el direccionamiento } \\
\text { estratégico y la articulación }\end{array}$ & $\begin{array}{l}\text { La mayoría de las respuestas presentan una relación regular debido a que la } \\
\text { distribución de frecuencia es uniforme }\end{array}$ \\
\hline $\begin{array}{l}\text { Evaluación sobre los sistemas integrados como único modelo de } \\
\text { gestión estructurado }\end{array}$ & $\begin{array}{l}\text { La mayoría de los entrevistados los considera regulares ya que las respuestas } \\
\text { presentan forma de distribución normal con promedio de } 2.62\end{array}$ \\
\hline $\begin{array}{l}\text { Evaluación sobre la contribución al mejoramiento continuo de la } \\
\text { institución }\end{array}$ & $\begin{array}{l}\text { La mayoría la considera entre deficiente y regular ya que las respuestas no } \\
\text { presentan forma de distribución normal }\end{array}$ \\
\hline Evaluación sobre la participación del personal en la implementación & Se puede decir que la mayoría la considera entre buena y excelente \\
\hline $\begin{array}{l}\text { Evaluación sobre el conocimiento de las metodologías de } \\
\text { implementación }\end{array}$ & La mayoría lo considera entre regular y bueno. \\
\hline Evaluación sobre la visión futura de la IES & La mayoría la considera entre buena y excelente \\
\hline Evaluación sobre los beneficios para la eficacia & La mayoría los considera entre deficientes y regulares \\
\hline Evaluación sobre la optimización de recursos & La mayoría la considera entre buena y excelente \\
\hline
\end{tabular}

Fuente: elaboración propia.

Con la existencia de sistemas de gestión que se implementan de forma separada las organizaciones enfrentan dificultades y duplicidad en los esfuerzos. Con la integración de estos sistemas se logra disminuir costos y simplificar las actividades y tareas a realizar gracias al trabajo en equipo y la eliminación de conceptos errados o que crean confusión. El sistema de gestión de calidad bajo el ambiente ISO 9001:2015 y el proceso de acreditación de programas académicos según los parámetros del CNA tienen una alta capacidad para consolidarse como un único sistema integrado bajo una sola autoridad con un solo bloque de responsabilidades, lo que hace más fácil la implementación, el seguimiento y el control.
No obstante, cabe mencionar que dentro de los inconvenientes presentados en la integración de sistemas de gestión de calidad están los cambios procedimentales que pueden incidir de manera importante en la organización y los individuos. Esto es más notorio cuando los sistemas se encuentran separados, sin duda el mayor obstáculo en la integración posiblemente debido a la falta de motivación de la dirección, la escasa conexión entre las actividades integradoras del sistema de gestión de la calidad por no contar con las competencias necesarias para lograrla o la ausencia de una metodología clara y eficiente para la integración (Ferguson et al., 2002). 
En cuanto a las IES, no está clara la metodología más apropiada para el logro de la mejora continua en sus procesos. Mientras que los sistemas de gestión de la calidad en las industrias manufactureras se analizan, evalúan y mejoran con base en las evidencias encontradas en sus procesos internos, los parámetros y requisitos de calidad en la educación superior colombiana están determinados por la reglamentación del Ministerio de Educación Nacional y el CNA, y por la percepción del servicio prestado por la IES. De allí que deba implementarse un sistema de gestión de la calidad que permita establecer estrategias ambiciosas y empodere a la institución haciéndola competitiva frente a las exigencias del mercado (Tlapa, 2009).

En la tabla 2 se presentan las etapas de la metodología resultante del presente estudio. La correlación expresada en la hoja de análisis propuesta por Juran (1990) se evidencia mediante un análisis vertical de la matriz de correlación que corresponde al nivel de concordancia de la norma ISO 9001:2015 con los parámetros establecidos por el CNA para la autoevaluación de programas académicos con fines de acreditación. Se puede observar que el numeral 10 (mejora) presenta el mayor valor de correlación, con un $80 \%$, en contraste con el numeral 6 (planificación), que con el $49.2 \%$ presenta el menor valor. Es importante mencionar también algunos otros valores sobresalientes, como los de los numerales 4 (contexto de la organización, 67.3\%), 7 (apoyo, 75.9\%), 9 (evaluación del desempeño, 62.7\%); y algunos valores medios, como los de los numerales 5 (liderazgo, $55.5 \%$ ) y 8 (operación, $56.8 \%$ ).

En cuanto el análisis vertical de correlación de la norma ISO 9001:2015 en Unitrópico, se observa que presenta un nivel alto de coherencia con los parámetros de autoevaluación con fines de acreditación del CNA ya que en promedio presenta un valor de correlación del $63.92 \%$. Así, se observa que la integración de los sistemas puede ser implementada de manera conjunta y eficiente.
Respecto al análisis horizontal de la matriz de correlación, que corresponde al nivel de concordancia de los parámetros establecidos por el CNA para la autoevaluación de programas académicos con fines de acreditación versus la norma ISO 9001:2015, se puede observar que el numeral 8 (organización, administración y gestión) presenta el mayor valor de correlación con un $83.5 \%$, en contraste con el numeral 1 (misión, proyecto institucional y de programa), con un valor del $52.7 \%$. Ahora bien, es importante mencionar algunos otros valores sobresalientes, como los de los numerales 2 (estudiantes, $64.7 \%$ ), 3 (profesores, 63.2\%), 4 (procesos académicos, $62.8 \%$ ), 5 (visibilidad nacional e internacional, 68.5\%), 7 (bienestar institucional, 78.1\%) y 9 (impacto de los egresados en el medio, 62.1\%), además de un valor medio en el caso del numeral 10 (recursos físicos y financieros, $56.1 \%$ ).

En cuanto al análisis horizontal, se observa un nivel alto de coherencia de los parámetros de autoevaluación con fines de acreditación del CNA en relación con la norma ISO 9001:2015 pues se presenta un valor promedio de correlación del $66.03 \%$. Por lo tanto, la integración de los sistemas puede ser implementada de manera conjunta y eficiente, tal y como lo sugiere el análisis vertical, aunque el nivel de correlación es ligeramente mejor.

Finalmente, se determinó que el nivel de correlación del sistema integrado de gestión de la calidad entre la norma ISO 9001:2015 y los parámetros de autoevaluación de programas con fines de acreditación del CNA en Unitrópico arrojó un porcentaje de correlación del 63,84\%. Ello evidencia un alto nivel de coherencia entre los dos sistemas de gestión, de modo que Unitrópico podrá implementarlos en conjunto para evitar la duplicidad de información, actividades, procesos y documentos y reducir tiempos y costos con base en la identificación de los aspectos comunes planteadas en la ciudadanía sistémica (Morín, 1999). 
Tabla 2. Articulación metodológica para la implementación

\begin{tabular}{|c|c|c|c|c|c|c|}
\hline $\begin{array}{l}\text { Etapas para la } \\
\text { aplicación }\end{array}$ & $\mathrm{N}^{\circ}\left(^{\circ}\right)$ & ISO 9001:2015 & $\begin{array}{c}\text { Correlación-hoja } \\
\text { de análisis }(\%)\end{array}$ & $\begin{array}{l}\mathrm{N} . \\
(*)\end{array}$ & Acreditación de programas-CNA & $\begin{array}{c}\text { Análisis hori- } \\
\text { zontal-matriz de } \\
\text { correlación }(\%)\end{array}$ \\
\hline Etapa 1 & 10 & Mejora & 80 & 8 & Organización, administración y gestión & 83.5 \\
\hline Etapa 2 & 7 & Apoyo & 75.9 & 7 & Bienestar institucional & 78.1 \\
\hline \multirow[b]{2}{*}{ Etapa 3} & \multirow[b]{2}{*}{4} & \multirow{2}{*}{$\begin{array}{l}\text { Contexto de la } \\
\text { organización }\end{array}$} & \multirow[b]{2}{*}{67.3} & 5 & Visibilidad nacional e internacional & 68.5 \\
\hline & & & & 6 & $\begin{array}{l}\text { Investigación y creación artística y } \\
\text { cultural }\end{array}$ & 68.5 \\
\hline \multirow{2}{*}{ Etapa 4} & \multirow{2}{*}{9} & \multirow{2}{*}{$\begin{array}{l}\text { Evaluación de } \\
\text { desempeño }\end{array}$} & \multirow{2}{*}{62.7} & 2 & Estudiantes & 64.7 \\
\hline & & & & 3 & Profesores & 63.2 \\
\hline \multirow{2}{*}{ Etapa 5} & \multirow[b]{2}{*}{8} & \multirow{2}{*}{ Operación } & \multirow{2}{*}{56.8} & 4 & Procesos académicos & 62.8 \\
\hline & & & & 9 & Impacto de los egresados en el medio & 62.1 \\
\hline Etapa 6 & 5 & Liderazgo & 55.5 & 10 & Recursos físicos y financieros & 56.1 \\
\hline Etapa 7 & 6 & Planificación & 49.2 & 1 & $\begin{array}{l}\text { Misión, visión y proyecto institucional } \\
\text { y de programa }\end{array}$ & 52.7 \\
\hline
\end{tabular}

Fuente: elaboración propia.

(*) Para ISO 9001:2015 se refiere al capítulo de requisitos de la estructura de alto nivel; para los lineamientos CNA, a los factores.

\section{CONCLUSIONES}

Se determinó la concordancia y coherencia de dos sistemas de gestión (ISO 9001:2015 y los parámetros de autoevaluación con fines de acreditación de programas del CNA) en la IES Unitrópico y se logró establecer una metodología para su integración, acorde a las necesidades institucionales, que aportará beneficios administrativos y académicos además de ofrecer un servicio de calidad en la formación del individuo. Se espera así impactar positivamente el desarrollo regional y nacional en términos de eficiencia en la gestión institucional, al lograr correlaciones superiores al $60 \%$ en los referenciales analizados.

La metodología propuesta, descrita en siete etapas, obedece a los conceptos de la ciudadanía sistémica de Morín (1999). La integración está fundamentada en los aspectos convergentes entre los dos sistemas de gestión.
Inicialmente expertos evaluaron la pertinencia del sistema integrado de gestión, el diagnóstico institucional de cada uno de los sistemas de manera individual y el análisis de correlación de los parámetros de cada sistema de gestión. Se determinaron así las posibilidades institucionales de implementar la metodología del sistema integrado de gestión.

Se evidenció que los parámetros de conocimiento, reconocimiento, ventajas, relación, estructuración, contribución a la mejora continua, participación del personal, metodología a aplicar, visión futura, beneficios y optimización del sistema integrado de gestión de la calidad obtuvieron un valor promedio ponderado de regular respuesta (con un valor cercano a 3). Ello determina un vago conocimiento e interés en relación con la norma de calidad ISO 9001:2015 y los lineamientos del CNA para 
acreditación de programas, además de un bajo criterio de empoderamiento y reconocimiento de la importancia de la implementación del sistema integrado de gestión. La institución deberá mejorar dicho aspecto con el fin de dar validez al proceso de integración.

Unitrópico, como IES, debe establecer políticas que le permitan crear conciencia en la comunidad académica y sus partes interesadas acerca de la importancia del sistema integrado de gestión de la calidad pues, aunque se tiene un conocimiento moderado de la norma NTC-ISO 9001:2015 y de los parámetros del CNA, se desconoce su importancia y las bondades de su implementación. Los cargos de dirección y decisión son los que deben estar seguros del aporte futuro de tal integración al cumplimiento de los objetivos de calidad, en alineación con el direccionamiento estratégico de la organización.

El análisis de correlación realizado en Unitrópico muestra una alta posibilidad de integración, con un valor promedio de $63.84 \%$ de elementos comunes entre los numerales de la norma NTC-ISO 9001:2015 y los parámetros del CNA. Por lo tanto, existe la posibilidad futura de lograr la acreditación de programas académicos en conjunto con la certificación de calidad.

Respecto al nivel de correlación analizando de manera individual la norma NTC-ISO 9001:2015 y los parámetros del CNA, ambos presentan una correlación importante que permite implementar y certificar cada uno de los sistemas, según el ente regulador que lo otorgue. Eso permite articularlos de manera conjunta y coherente en pos de la mejora continua de los procesos académicos y administrativos de Unitrópico establecidos según los planes de mejora para cada sistema de gestión.

La metodología propuesta para el sistema integrado de gestión de la norma NTC-ISO 9001:2005 y los parámetros del CNA permite corroborar la hipótesis planteada, según la cual al implementar la metodología en Unitrópico se podrán articular los dos sistemas en función de hacer eficientes los procesos académicos y administrativos.

Aunque Unitrópico deberá mejorar sustancialmente en la implementación de los sistemas de gestión propuestos en este estudio, debe enfocarse especialmente en el levantamiento de información, la caracterización, la evaluación y la mejora de sus procesos incentivando el empoderamiento y el liderazgo de las partes interesadas. Especialmente la dirección, que deberá encabezar el desarrollo y apoyo al sistema integrado de gestión, ademas de realizar inversiones en infraestructura, equipos, laboratorios, escenarios de formación y granjas experimentales.

Es importante tener en cuenta que el análisis de la integración de la norma ISO 9001:2015 y los parámetros de acreditación del CNA puede llegar a ser una metodología aplicable en otras IES. En efecto, la correlación encontrada en este estudio muestra que en general tales sistemas pueden operar de manera conjunta gracias a que en gran medida son mutuamente incluyentes.

Queda pendiente realizar una validación de la metodología para crear un modelo de integración que pueda ser usado en instituciones universitarias de carácter privado. Además, es importante realizar el estudio en IES de carácter público incluyendo comparativos, con el fin de establecer un modelo universal aplicable a cualquier IES.

Los procesos de autoevaluación y diagnóstico de la norma ISO 9001:2015 serán importantes referentes para iniciar el proceso de integración del sistema de gestión de la calidad pues otorgan una línea de base para empezar la planificación asertiva, la toma de conciencia en la comunidad universitaria y el empoderamiento del sistema de gestión. Además, es importante hacer seguimiento y control para la mejora continua. 
Unitrópico podrá implementar la metodología de integración del sistema de gestión de la calidad aprovechando sus recursos humano y material y su infraestructura, y mediante una clara posición frente a la calidad educativa. La dirección deberá fortalecer los procesos académicos y administrativos en función de la mejora continua y el posicionamiento de la institución educativa en el contexto regional y nacional.

\section{REFERENCIAS}

CNA (Consejo Nacional de Acreditación) (enero, 2013). Lineamientos para la acreditación de programas de pregrado. Bogotá: CNA. Recuperado de https://tinyurl. $\mathrm{com} / \mathrm{y} 5 \mathrm{f} 4 \mathrm{yq} 4 \mathrm{q}$

Espín, J., Jiménez, D. y Costa, M. (2014). La gestión de calidad: importancia de la cultura organizativa para el desarrollo de variables intangibles. Revista Europea de Dirección y Economía de la Empresa, 23(3), 115-126. Doi: https://doi.org/10.1016/j.redee.2014.02.002

Ferguson, M., García, M. y Bornay, M. (2002). Modelos de implantación de los sistemas integrados de gestión de la calidad, el medio ambiente y la seguridad. Investigaciones Europeas de Dirección y Economía de la Empresa, 8(1), 97-118.

Hendricks, K. y Singhal, V. (1996). Quality awards and the market value of the firm: An empirical investigation. Management Science, 42(3), 415-436. Doi: https://doi.org/10.1287/mnsc.42.3.415

Hernández, R., Fernández, C. y Baptista, P. (2014). Metodología de la investigación. México: McGraw-Hill Education.
Icontec (Instituto Colombiano de Normas Técnicas y Certificación) (2015). Norma Técnica Colombiana NTC-ISO 9001. Sistemas de gestión de la calidad. Bogotá: Icontec.

Juran, J. (1990). Juran y la planificación para la calidad. Madrid: Díaz de Santos.

Karapetrovic, S. y Willborn, W. (1998). Integration of quality and environmental management systems. The TQM Magasine, 10(3), 204-213. Doi: https://doi.org/10.1108/09544789810214800

Kumar, V., Choisne, F., De Grosbois, D. y Kumar, U. (2009). Impact of TQM on company's performance. International Journal of Quality \& Reliability Management, 26(1), 23-37. Doi: https://doi. org/10.1108/02656710910924152

López, J. (2014). El sistema de gestión integrado. Bogotá: Icontec.

Morduchowicz, A. y Arango, A. (2007). Gobernabilidad, gobernansa y educación en Argentina. Buenos Aires: Unesco.

Morín, E. (1999). Los siete saberes necesarios para la educación del futuro. París: Unesco.

Pérez, Z. (2011). Los diseños de método mixto en la investigación en educación. Una experiencia concreta. Revista Electrónica Educare, 15(1), 15-29.

Powell, T. (1995). Total quality management as competitive advantage: A review and empirical study. Strategic Management Journal, 16(1), 15-37. Doi: https://doi.org/10.1002/smj.4250160105 
Quintero, M. P. (2007). Validación y aplicación de la entrevista semiestructurada codificada y observación a la idoneidad del profesor, en el segundo año de Ciencias de la Salud. Revista de Educación en Ciencias de la Salud, 6(2), 93-100.

Rahman, S. y Bullock, P. (2005). Soft TQM, hard TQM, and organizational performance relationships: An empirical investigation. Omega, 33(1), 73-83. Doi: https://doi.org/10.1016/j.omega.2004.03.008

Ruiz, M, Borboa, M. y Rodríguez, J. (2013). El enfoque mixto de investigación en los estudios fiscales. Tlatemoani. 13, 1-25.

Tapia, F. (2015). Clima organizacional en una institución de educación superior mexicana. Revista Colombiana de Salud Ocupacional, 3(5), 11-17.

Tlapa, D. (2009). Gestión de la calidad y del medio ambiente en instituciones de educación superior mediante integración de ISO 9001 e ISO 14001. Formación universitaria, 2(2), 35-46. Doi: https:// doi.org/10.4067/S0718-50062009000200006
Unitrópico (2011). Proyecto educativo institucional "Investigando la Orinoquia colombiana. Recuperado de https://drive.google.com/file/d/1ehq535frf4uE Os2CEyfG0NIM9sKRyiad/view

Unitrópico, Consejo Superior (29 de agosto de 2011). Acuerdo 74. Por medio del cual se adoptan y reglamentan las modalidades de trabajo de grado para los programas de pregrado de la Fundación Universitaria Internacional del Trópico AmericanoUnitrópico. Secretaría General, Yopal.

Unitrópico (2014). Informe final autoevaluación. Yopal: Unitrópico.

Univalle (s. f.). Herramienta de diagnóstico ISO 9001:2008 [online]. Cali: Univalle. Recuperado de https://tinyurl.com/y379dqxn

Wigodski, J. (8 de junio de 2010). Fuentes primarias y secundarias [entrada de blog]. Recuperado de http://metodologiaeninvestigacion.blogspot.com. co/2010/07/fuentes-primarias-y-secundarias.html 